\title{
UNE MĖRE ET SES DEUX FILLES JUMELLES UNIVITELLINES ATTEINTES DE CHONDRODYSPLASIE
}

\author{
par \\ U. Pfändler \\ La Chaux-de-Fonds (Suisse)
}

\section{Introduction}

La différenciation des multiples formes de nanisme proportionné ou dysproportionné, est loin d'être achevée. Les temps ne sont pas si lointains, où l'on confondait d'autres types dysproportionnés avec la Chondrodysplasie classique et sa symptomatologie caractéristique. Anatomo-pathologiquement, nous savons qu'elle manifeste une formation primaire insuffisante du cartilage, aboutissant à l'arrêt prématuré de l'ossification enchondrale. Par contre l'ossification périostale est généralement normale. A la dysplasie cartilagineuse s'ajoute la perturbation dans le développement de différents tissus dérivés du mésenchyme.

L'observation de nombreuses souches a permis d'établir le conditionnement héréditaire et monogénique de la chondrodysplasie. Si l'expressivité de l'atteinte peut varier, sa pénétrance, par contre, est pratiquement totale. Comme la tare se transmet par voie dominante, les enfants atteints auront nécessairement un parent achondroplase. Si tel n'est pas le cas, nous devons admettre qu'il s'agit d'une nouvelle mutation survenue dans un gamète de l'un des parents. C'est d'ailleurs cette notion qui a permis d'évaluer, sur la base d'un matériel étendu, le taux de mutation du gène morbide en question.

Il s'est avéré que certaines formes de nanisme dysproportionné que l'on avait autrefois rattachées à la chondrodysplasie, suivaient le mode récessif simple. Leur conditionnement génétique différent confirma d'emblée que l'on était en présence d'entités nosologiques distinctes. C'est le cas, par exemple, pour le nanisme de Morquio et pour la Dysostosis multiplex (syndrome de Hurler).

La chondrodysplasie étant essentiellement héréditaire et sa pénétrance pratiquement totale, il serait intéressant de le confirmer par l'étude des jumeaux. Si nos conceptions sont exactes, toutes les paires univitellines seraient nécessairement concordantes, pour autant qu'une phénocopie ne puisse pas occasionnellement aboutir à des manifestations identiques.

L'étude des jumeaux achondroplases publiés dans la littérature, se heurte essentiellement à deux difficultés:

1. Dans les anciennes publications, les critères établissant avec certitude la monozy. gotie, font souvent défaut. 
2. L'absence d'une classification adéquate des différents types de nanisme dysproportionné, eut pour effet d'assimiler à la chondrodysplasie, des cas certains de Morquio ou des formes abortives de Hurler.

Nous disposons aujourd'hui d'un très petit nombre de paires mono - ou dizygotiques, manifestant une chondrodysplasie certaine. Pour les univitellins concordants, l'on cite respectivement les paires de Nonne, de Tack et de Goodman. Pour les cas décrits par Lommel et par Schemensky, il n'est pas possible d'exclure la dizygotie.

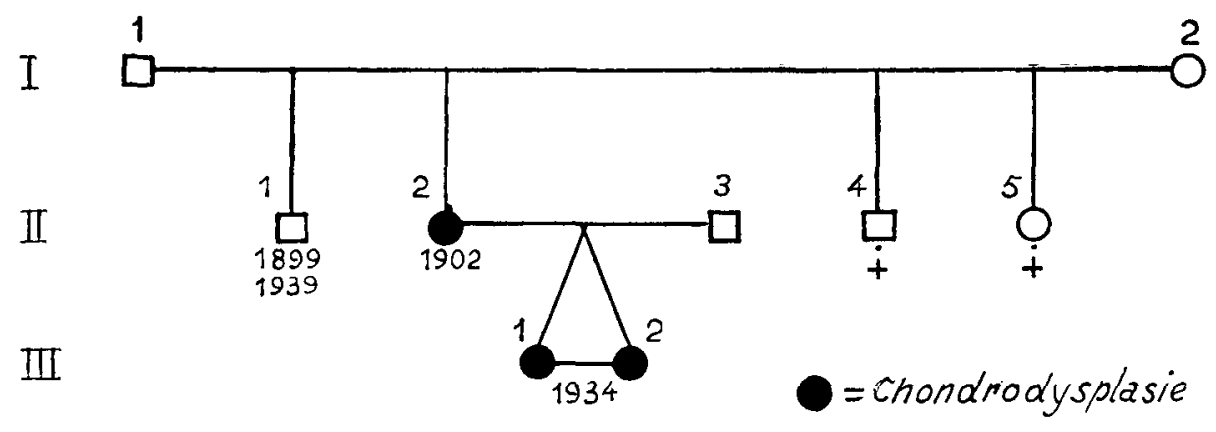

Fig. 1. Tableau généalogique

Plus nombreux sont les jumeaux bivitellins discordants, notamment ceux décrits par Peralta Ramos et Murtagh (une fille atteinte, un frère sain), par Hutchinson (une sœur atteinte, un frère sain), par Klein, par Parphon, Shunda et Zalplachta, par Rischbieth et Barrington (une sœur atteinte, un frère sain), par Lepage (un couple gémellaire dont la sœur est atteinte), par Warner (deux frères dont un atteint), et par Pritchard (cas atypique de chondrodysplasie).

Romberg a observé un couple gémellaire concordant.

Baumm, Neale et Hucknall ont aussi signalé la présence d'achondroplasie chez des jumeaux.

Benjamin et Brookner avaient admis la monozygotie pour leur paire discordante, mais leurs examens de concordance des caractères sont trop incomplets pour que l'on puisse exclure la dizygotie. Etant donné la pénétrance totale de la chondrodysplasie, une discordance absolue des monozygotiques nous obligerait à admettre l'existence de phénocopies achondroplases.

\section{Observation personnelle}

Nous avons examiné dans les environs de Bienne (Suisse), une mère et ses deux filles jumelles monozygotiques, atteintes toutes les trois de Chondrodysplasie (fig. 1). Un frère et une sœur de la mère sont décédés respectivement à 3 semaines et à 3 jours.

1. La mère (II/2), née en 1902 (voir fig. 2) aurait fait une méningite compliquée de pneumonie et pleurésie à 13 ans. Pas de fausse-couche. En 1934 elle subit une césarienne, et en 1948 une hystérectomie pour fibrome utérin. 
Caractères particuliers:

Patiente de petite taille, mesurant $127 \mathrm{~cm}$.

Poids: $41 \mathrm{~kg} .150$.

Périmètre maximal de la tête: $62 \mathrm{~cm}$.

Périmètre horizontal de la tête: $55 \mathrm{~cm}$.

Couleur des yeux: gris-foncé.

Couleur des cheveux: châtain foncé.

Couleur de la peau: blanc-rosé.

Forme des cheveux: plats.

$\mathrm{Nez}$ et oreilles: bien conformsé.

Lobule de l'oreille: libre.

Pilosité digitale: néant.

Handedness: droitière.

Anomalies des ongles: plats.

PTC: +++ .

2. Dans l'anamnèse des deux filles jumelles, Gertrude (III/1) et Jda (III/2), nées en 1934, et extraites par césarienne d'une mère achondroplase, on retient les faits suivants:

Gertrude (III/1): coqueluche, pneumonie, rougeole en bas-âge. Comme petite fille, eczéma aigu suintant, ayant duré 3 semaines. Appendicectomie en 1948. Règles normales, début à 12 ans.

$J d a(I I I / 2)$ : coqueluche, pneumonie, rougeole en bas-âge. Appendicectomie en 1942, oreillons en 1946, abcès du sein en 1951. Règles normales début à 13 ans.

Dans leurs status, les deux sœurs présentent les caractères suivants (fig. 3, 4, 5. 6a, $6 b$, et tableaux 7 et 8 ):

Tableau N. 7

\begin{tabular}{|c|c|c|}
\hline & Gertrude, III/1 & $\mathrm{Jda}, \mathrm{III} / 2$ \\
\hline Taille . . . . . . . . . . . . . . & $131 \mathrm{~cm}$. & $130 \mathrm{~cm}$. \\
\hline Poids . . . . . . . . . . . . & $41 \mathrm{~kg}$. & $41 \mathrm{~kg} .800$ \\
\hline Circonférence du thorax . . . . . . . . . & $81 \mathrm{~cm}$. & $83 \mathrm{~cm}$. \\
\hline Circonférence de l'abdomen .......... & $68 \mathrm{~cm}$. & $69 \mathrm{~cm}$. \\
\hline Périmètre maximal de la tête . . . . . . . & $64 \mathrm{~cm}$. & $64 \mathrm{~cm}$. \\
\hline Périmètre horizontal de la tête ........ & $53,5 \mathrm{~cm}$. & $54 \mathrm{~cm}$. \\
\hline $\begin{array}{l}\text { Distance Spina iliaca anterior superior - interligne } \\
\text { articulaire externe du genou ......... }\end{array}$ & $\begin{aligned} & \text { dr. } 32 \mathrm{~cm} \\
& \text { g. } 32 \mathrm{~cm}\end{aligned}$ & $\begin{array}{l}32 \mathrm{~cm} \\
32 \mathrm{~cm}\end{array}$ \\
\hline 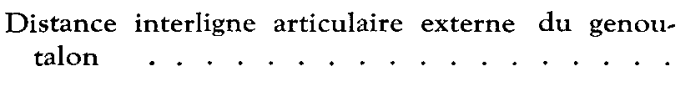 & $\begin{array}{l}\text { dr. } 35 \mathrm{~cm} . \\
\text { g. } 34,5 \mathrm{~cm} .\end{array}$ & $\begin{array}{l}34,5 \mathrm{~cm} \\
35 \mathrm{~cm}\end{array}$ \\
\hline Distance processus coracoideus - olecranon. & $\begin{array}{l}\text { dr. } 25 \mathrm{~cm} . \\
\text { g. } 25 \mathrm{~cm} .\end{array}$ & $\begin{array}{l}27 \mathrm{~cm} . \\
27 \mathrm{~cm}\end{array}$ \\
\hline Distance olecranon - articulation de la main. & $\left\{\begin{array}{r}\text { dr. } 19 \mathrm{~cm} \\
\text { g. } 19 \mathrm{~cm}\end{array}\right.$ & $\begin{array}{l}19 \mathrm{~cm} \\
19 \mathrm{~cm}\end{array}$ \\
\hline
\end{tabular}




\section{U. Pfändler: Une mère et ses deux filles jumelles univitellines, etc.}

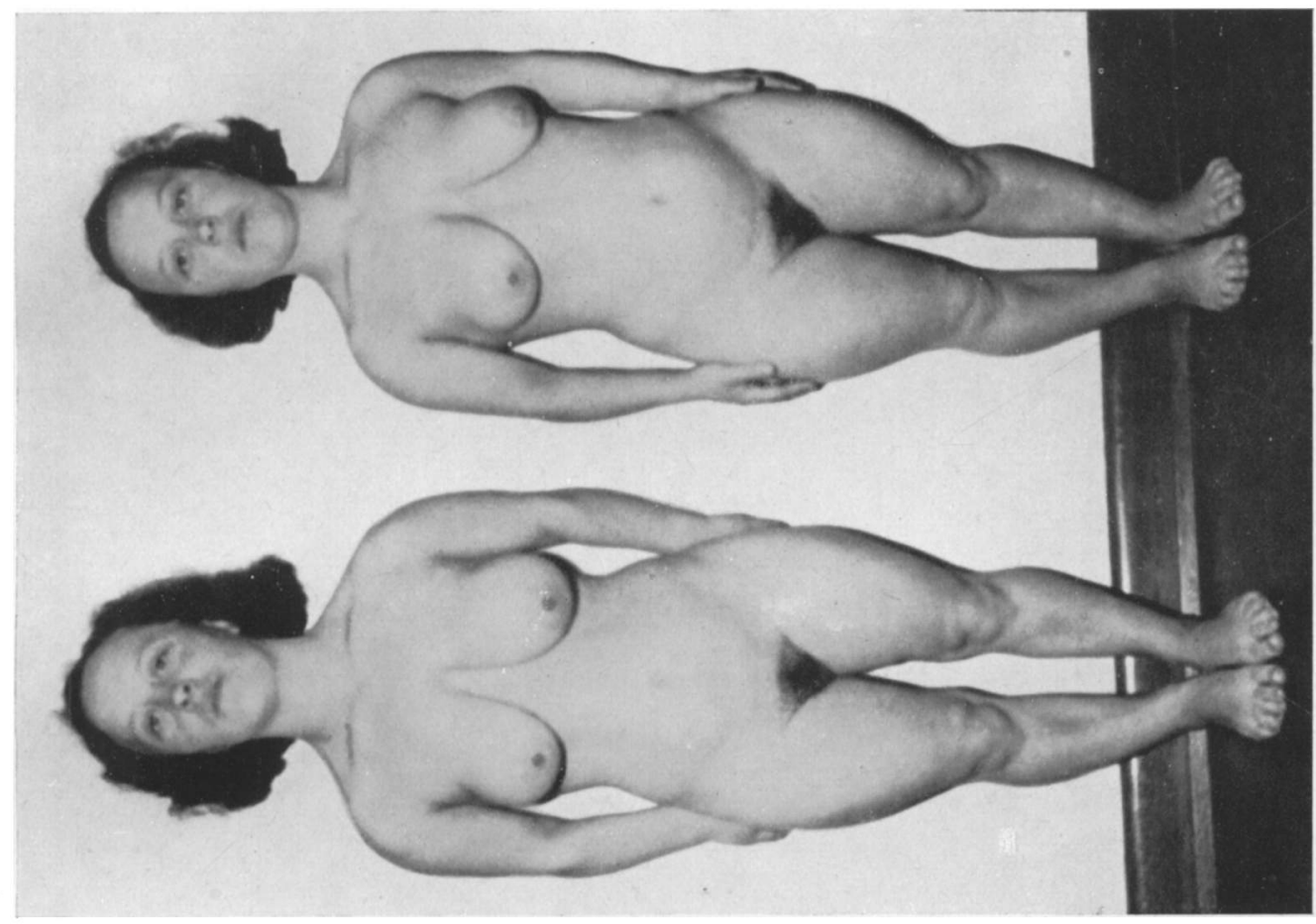

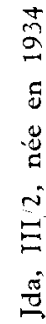

$m$

$\dot{m}$

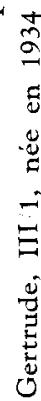

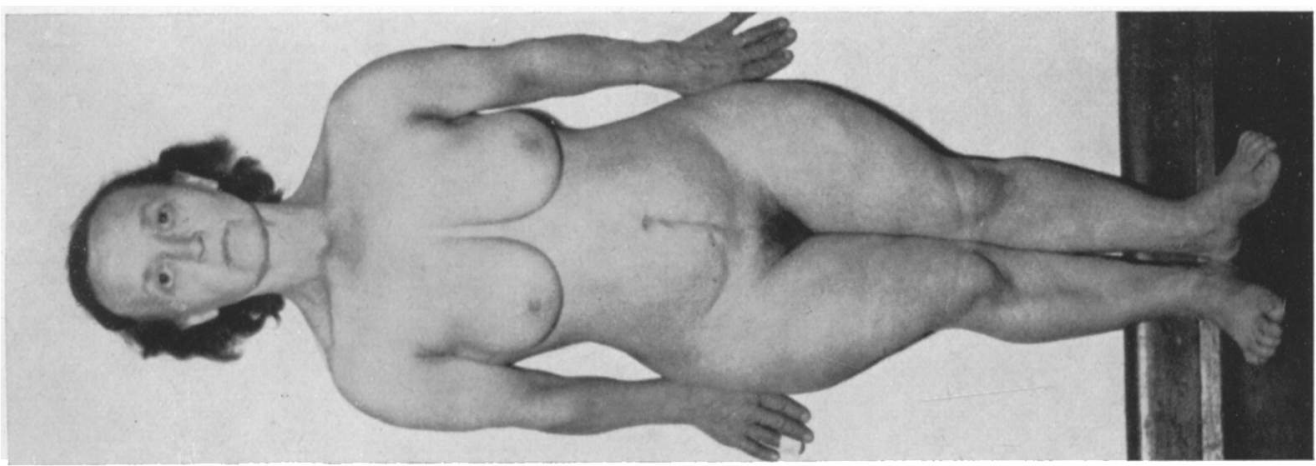

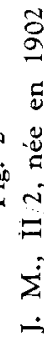


Tableau N. 8

\begin{tabular}{|c|c|c|c|c|c|c|c|c|c|}
\hline Caractèr & ère & & & & & & Gertrude, III/1 & Jda, III $/ 2$ & Concordant \\
\hline . . . . . . . & . . . & & . . & . & . & . & féminin & féminin & + \\
\hline Groupe sanguin . . & . . & . & . . & . & . & . & A & A & + \\
\hline Facteurs MN . . . & . . & . & . & . & . & . & $\mathrm{MN}$ & $\mathrm{MN}$ & + \\
\hline Facteurs Rhésus & . & . & . . & . & . & . & $\mathrm{CDe} / \mathrm{cDE}$ & $\mathrm{CDe} / \mathrm{cDE}$ & + \\
\hline Couleur des yeux . & . . & . & . & . & . & . & gris-vert & gris-vert & + \\
\hline Structure de l'iris & . . & . & $\cdot \cdot$ & . & - & • & . . . . . & . . . . & + \\
\hline $\begin{array}{c}\text { Disposition des vaisse } \\
\text { de l'oeil }\end{array}$ & eaux & du & fon & & • & &.$\cdot$. & . . & + \\
\hline Astigmatisme bilatéral & 1 dire & ect, & 0,5 & . & . & . & + & + & + \\
\hline Naevus sur l'iris & . & · & $\cdot \cdot$ & . & . & • & présent à l'œil g. & présent à l'oeil dr. & + \\
\hline Couleur des cheveux & . & • & . . & . & • & . & châtain & châtain & + \\
\hline Couleur de la peau & . & . & . $\cdot$ & . & . & . & blanc-rosé & blanc-rosé & + \\
\hline Forme des cheveux & . & . & . & . & . & . & frisé & frisé & + \\
\hline Forme des sourcils & . & . & . & - & . & . & 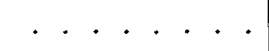 & $\cdot \cdot$ & + \\
\hline Forme du nez & . & . & . & . & - & . & . . . . . . & . . . . . . & + \\
\hline Taille des oreilles & . . & . & . . & . & . & . & $6 \mathrm{~cm}$. (dr. et g.) & $6 \mathrm{~cm}$. (dr. et g.) & + \\
\hline Lobule de l'oreille & . . & . & . . & . & . & . & soudé & soudé & + \\
\hline Forme des lèvres & . & . & . & - & · & . & . . . . . . . & . . . & + \\
\hline Plis de la langue & . & 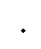 & - & . & - & - & . . . . . . . & . . . . & + \\
\hline Lentigines . . . . & . . & . & . & . & . & . & présents & présents & + \\
\hline Tourbillon du cuir ch & hevel & & & - & - & . & absent & absent & + \\
\hline Pilosité digitale . . & . $\cdot$ & $\cdot$ & $\cdot \cdot$ & - & - & • & absente & absente & + \\
\hline Handedness $\quad \cdot \cdot$ & $\cdot$. & . & . . & • & - & . & droitière & droitière & + \\
\hline PTC . . . . . & $\therefore$ & . & . & . & . & . & +++ & + & + \\
\hline Forme du bassin & . $\cdot$ & . & . . & . & . & & . . . . . . . & . . . . . . . . & + \\
\hline
\end{tabular}

Les examens oculaires (Dr. P. Zwahlen) donnent lieu aux observations suivantes:

a) Gertrude, III/1: un naevus sur l'iris à l'œil gauche à $2 \mathrm{~h}$. Les cryptes iriennes ne sont pas très marquées. Les anneaux périphériques concentriques, par contre, sont très nets. Un bord pigmentaire pupillaire, petit et très régulier, sans flocculi. Javal: aux deux yeux, astigmatisme direct de 0,5. Couleur de l'iris: gris-vert. Légère exophtalmie droite.

Fond de l'oil droit: anneau atrophique avec bord pigmentaire. Fond de l'œil gauche: anneau pigmentaire seul, avec un ancien foyer de choriö̈dite au bord de la papille, vers dix h.

Vision: œil droit: 1, un peu difficilement avec sphérique $+1,25$. Oeil gauche: 1 , avec sphérique $+1,25$.

b) $J d a$, III/2: même structure anatomique de l'iris que Gertrude. On retrouve un naevus de la même étendue, mais à l'œil droit. Un bord pigmentaire pupillaire petit et 


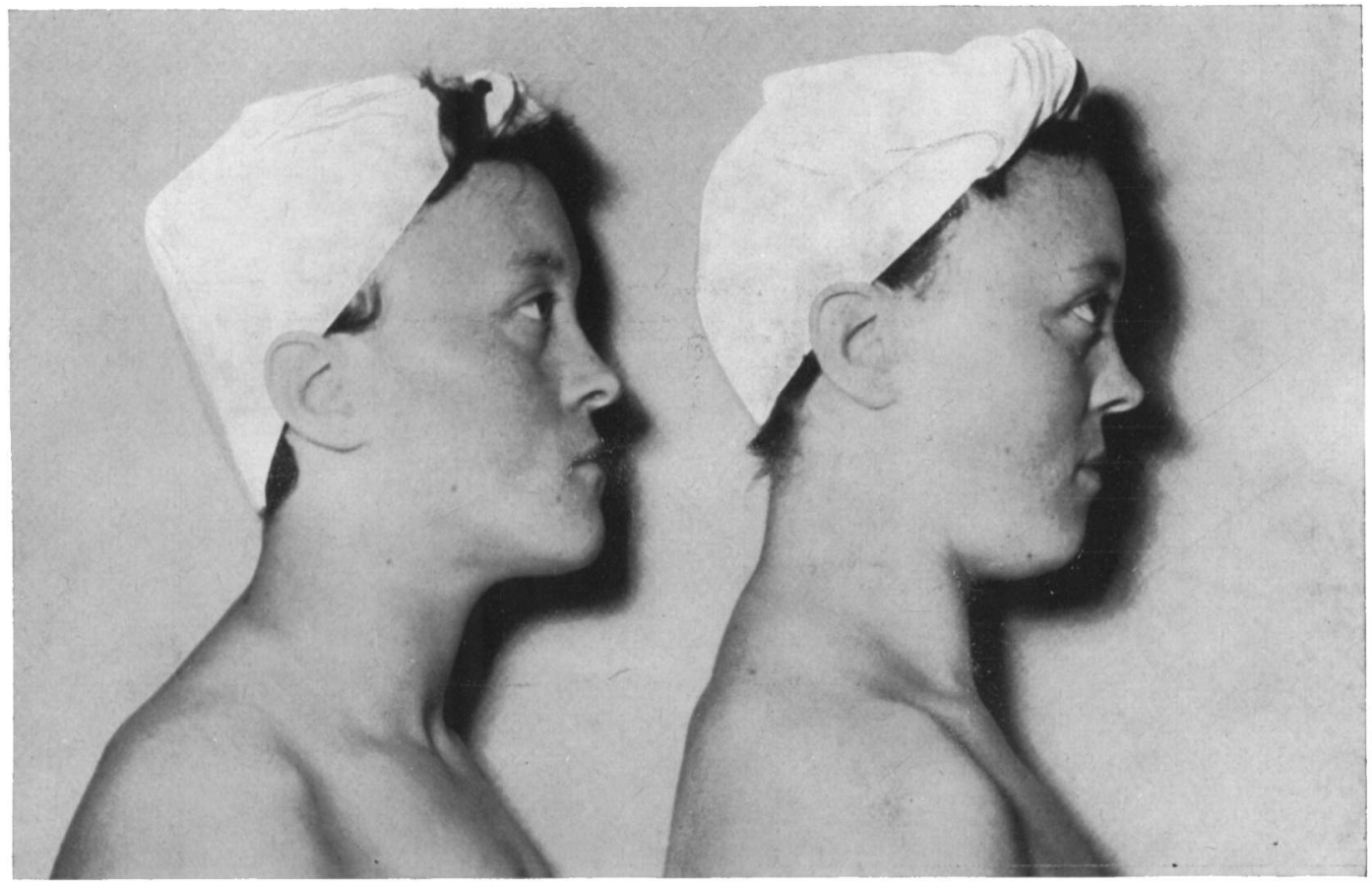

Gertrude, III/1

Fig. 4. profil droit

Jda, III 2

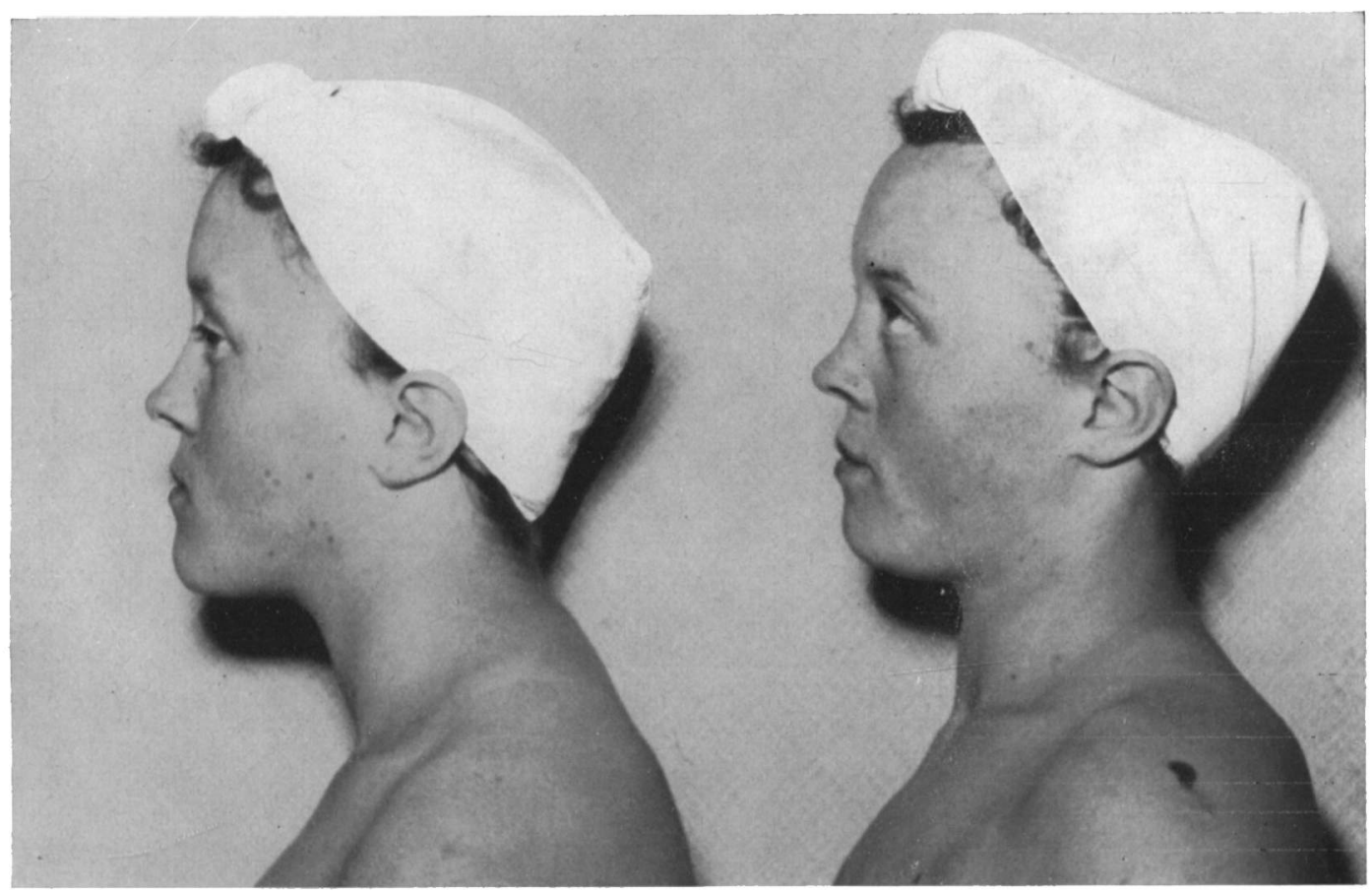

Gertrude, III/1

Fig. 5. profil gauche

$\mathrm{Jda}, \mathrm{III} / 2$ 

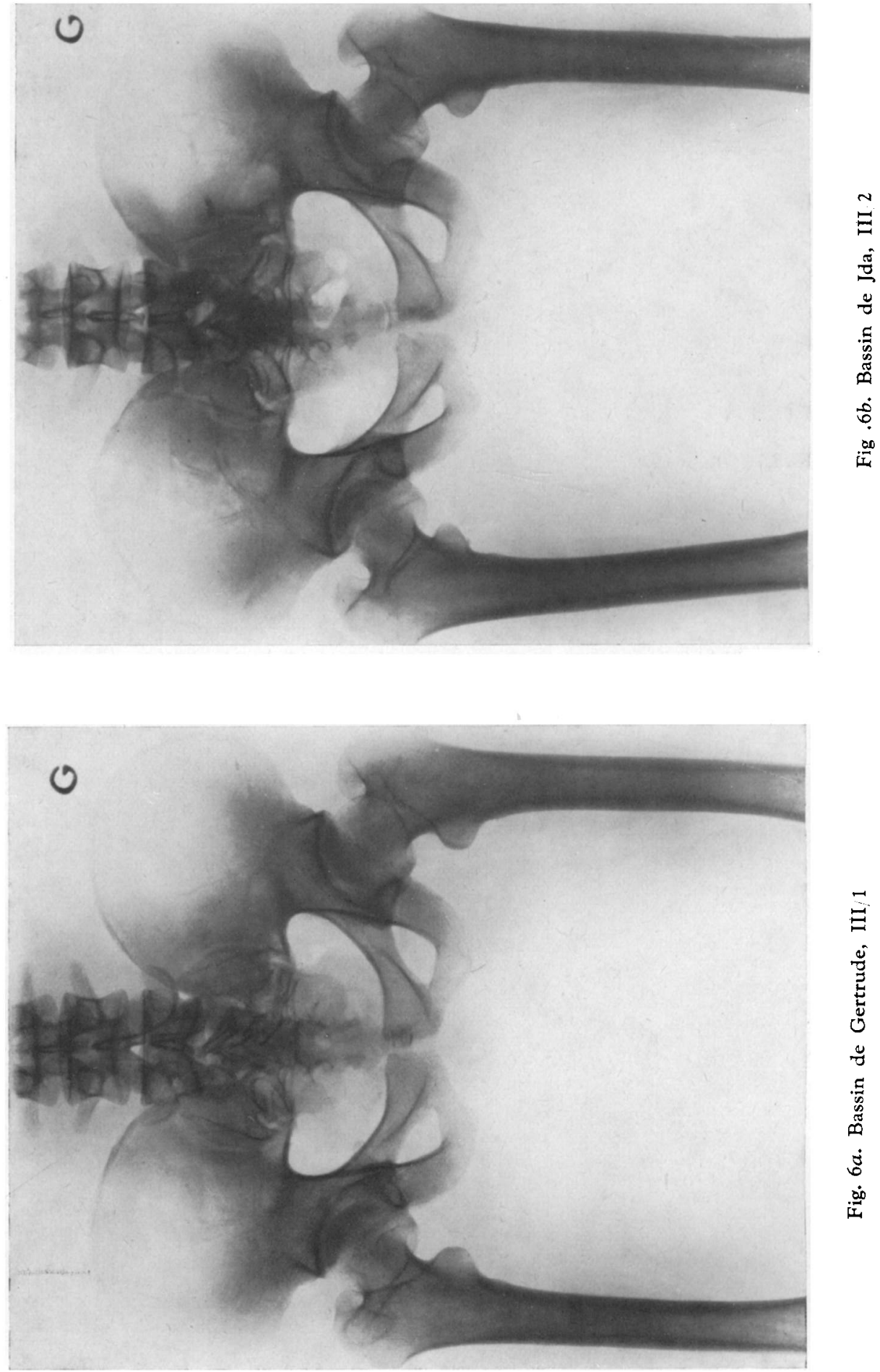
très régulier, sans flocculi. Javal: aux deux yeux, astigmatisme direct de 0,5. Couleur de l'iris: gris-vert, semblable à celle de Gertrude. Les pupilles sont bien rondes, égales. Légère exophtalmie droite.

Fond de l'œil droit: anneau pigmentaire, peu marqué.

Fond de l'œil gauche: anneau atrophique avec bord pigmentaire, peu marqué.

Vision: œil droit: 1,25 avec sphérique $+1,25$. Oeil gauche: compte les doigts à $30 \mathrm{~cm}$. (amblyopie congénitale). Strabisme convergent, concomitant, latent, amblyopique.

\section{Conclusion}

Il ne fait aucun doute que nous soyons ici en présence d'une paire gémellaire univitelline, concordante pour la chondrodysplasie. Les deux filles ont reçu le gène de leur mère achondroplase (II/2). Tous les ascendants directs de cette dernière sont de constitution normale. Nous devons donc admettre qu'une nouvelle mutation, responsable de la tare, s'est produite dans un gamète de l'un ou l'autre de ses parents.

\section{Bibliographie}

Benjamin, E. L. \& Brookner: A., J. Pediat. 4, 352, 1934.

Bracher, M.: Ztschr. f. orthop. Chir. 1932, 503.

Gedda, L.: Studio dei Gemelli, Chap. 12, p. 630-632, Ed. Orizzonte Medico, Roma 1951.

Goodman, S. Y.: Ohio St. M. J. 41, 521, 1945.

Hutchinson, R.: Proc. Roy. Soc. Med. 3, 41, 1910.

Klein, A.: Zbl. Path. 12, 1901.

Lepage: Compt. rend Soc. d'Obst., de Gynéc. et de Péd. 6, 270, 1904.

Neale, A. W. \& Hucknall, R. H.: Arch. Dis. Childr. 9, 51, 1934.i

Parphon, Shunda \& Zalplachta: Nouv. iconogr. Salpêtrière, 18, 539, 1905.

Peralta Ramos, A. \& Murtagh, J. J.: Dia med. 10, 764, 1938.

Pritchard, E.: Brit. J. Child. Dis. 25, 101, 1928 (extrait dans: Brit. J. Radiol. 1, 205, 1928).

Rischbieth \& Barrington: Eugenics Lab. Mem. London. 15, 1912.

Romberg, M.: De rachitide congenita, Diss. Berlin, 1817.

SCHEMENSKY: Ztschr. f. Röntgenkde, 15, 385, 1912.

WARNER, A.: Brit. M. J. 1928, 983 (3543).

Weygandt: Dans Gütt, Handb. d. Erbkrankh. 6, 188, 1940; EckHaRDr, H.: Körperliche Missbildungen.

Nous exprimons notre vive gratitude à M.le Prof. Grumbach, de l'Institut d'Hygiène de Zurich, qui a bien voulu se charger de la détermination des facteurs sanguins.

\section{Résumé}

Les paires gémellaires concordantes ou discordantes pour la chondrodysplasie, sont encore peu nombreuses. Nous décrivons une famille dans laquelle la mère et ses deux filles jumelles sont atteintes de la maladie. La monozygotie des sœurs est absolument certaine. 
SOMMARIO

Le coppie gemellari concordanti o discordanti rispetto alla condrodisplasia sono ancora poco numerose. Noi descriviamo una famiglia nella quale la madre e le sue due figlie gemelle sono colpite dalla malattia. La monozigotia delle sorelle è assolutamente certa.

\section{SUMMARY}

Till to-day there are very few concordant and discordant twin pairs known with regard to chondrodysplasy. We describe here a family in which as well the mother as also her both twin daughters are affected with this disturbance of development. The monozygosity of the sisters is beyond all doubt.

\section{ZUSAMMENFASSUNG}

Es sind bis heute sehr wenige, in Bezug auf Chondrodysplasie konkordante oder diskordante Zwillingspaare bekannt. Wir beschreiben hier eine $\mathrm{Fa}$ milie, in welcher sowohl die Mutter als auch ihre beiden Zwillingstöchter, mit dieser Entwicklungsstörung behaftet sind. Die Eineiigkeit der Schwestern steht ausser Zweifel. 